\title{
ILCEA
}

Revue de l'Institut des langues et cultures

d'Europe, Amérique, Afrique, Asie et Australie

3 | 2002

Le facteur culturel dans la traduction des textes

pragmatiques

\section{Le casse-tête de la traduction du mot « gender » en français}

Josiane Hay

\section{(2) OpenEdition}

Journals

Édition électronique

URL : http://journals.openedition.org/ilcea/832

DOI : 10.4000/ilcea.832

ISSN : 2101-0609

Éditeur

UGA Éditions/Université Grenoble Alpes

Édition imprimée

Date de publication : 15 juin 2002

Pagination : 113-126

ISBN : 2-9515849-0-3

ISSN : 1639-6073

Référence électronique

Josiane Hay, "Le casse-tête de la traduction du mot « gender » en français », ILCEA [En ligne], 3 | 2002, mis en ligne le 08 juin 2010, consulté le 30 avril 2019. URL : http://journals.openedition.org/ilcea/832 ; DOI : $10.4000 /$ ilcea. 832 


\title{
Le casse-tête de la traduction du mot «gender» en français
}

\author{
Josiane Hay \\ Université Joseph Fourier \\ Université Stendhal-Grenoble 3, GREMUTS
}

\section{Présentation du problème}

Le terme gender est fréquent dans les textes actuels de sociologie ou touchant aux questions de société en général et sa traduction constitue souvent, avec quelques autres termes tels que community ou stigma, un véritable casse-tête. Lorsqu'il apparaît de manière isolée, il est relativement facile de lui trouver un équivalent de type «sexe» ou «différenciation homme-femme», mais lorsque le terme est récurrent, ou que l'argumentation du texte se fonde sur lui, le problème de sa traduction (ou de sa non-traduction) se pose avec acuité. Le présent article illustre une situation de ce type.

Les difficultés évoquées ici se sont posées au cours de la traduction d'une publication du Département Santé et développement de l'enfant et de l'adolescent de l'Organisation mondiale de la santé et ONUSIDA. Il s'agissait de deux versions d'un même document consacré aux jeunes garçons et aux garçons adolescents: une version courte présentée sous forme d'un petit livret de 8 pages (4 pages recto verso format A4 plus la couverture) intitulée Boys in the Picture, et une version longue de 40 pages de texte (20 pages recto verso format A4) intitulée What about boys? L'objectif de cette publication est de sensibiliser les décideurs, travailleurs sociaux, personnels médicaux, etc. aux problèmes que rencontrent les garçons à la sortie de l'enfance et à l'adolescence. L'approche utilisée fait appel aux outils de la sociologie et du féminisme américains. La publication est destinée à un public francophone. La plupart des pays cités dans l'étude sont des pays en développement.

La lecture du document fait naître une première interrogation d'ordre culturel, dont on ne peut faire l'économie avant de se lancer dans la traduction: quelle est l'adéquation entre l'approche choisie et les objectifs recherchés. La plupart des pays francophones hors de l'Europe et de l'Amérique du Nord sont situés en Afrique. Une approche sociologique et féministe américaine convient-elle pour ces pays? L'original étant en anglais, la même question peut se poser pour les pays d'Afrique 
anglophone. Comprendront-ils mieux l'approche choisie que les pays francophones? La question concerne le traducteur puisque celui-ci doit décider, compte tenu des destinataires, de la distance à prendre avec la terminologie de l'approche choisie par l'auteur. La traduction d'un texte féministe destiné à un marché francophone essentiellement occidental exige tout naturellement un respect fidèle des concepts et de leur expression et une recherche terminologique pouvant aller jusqu'à l'invention de néologismes adaptés. Dans un texte potentiellement destiné à tous les pays membres d'une organisation internationale, développés ou non, francophones ou non, jusqu'où le traducteur doit-il aller dans le respect de la terminologie? On peut choisir de respecter fidèlement l'approche choisie par l'auteur anglophone et avoir recours à tous les néologismes disponibles. On peut aussi tenter d'utiliser une terminologie plus classique, plus accessible, mais au risque de trahir l'approche, de l'édulcorer. Un des problèmes de cette traduction a donc été de trouver une voie moyenne cohérente entre une réécriture du texte à l'aide d'une terminologie classique et le respect total de l'approche. La tension entre les deux pôles a été particulièrement forte dans le cas de gender et ses diverses combinaisons et collocations, problème qui sera traité ici, mais d'autres problèmes lexicaux liés à l'approche choisie se sont posés, sur lesquels je reviendrai à la fin du présent article.

\section{Gender}

Selon le dictionnaire Twentieth Century Words de John Ayto (OUP, 1999) le terme gender dans le sens:

The sex of a human being, from the point of view of the social and cultural, as opposed to the biological, distinctions between the sexes. Often used as a noun.

date de l'année 1963. Ce dictionnaire cite Alex Comfort:

The gender role learned by the age of two years is for most individuals almost irreversible, even if it runs counter to the physical sex of the subject. (p. 408).

La troisième édition du Shorter Oxford donne, sous gender (éd. de 1973):

1. kinds 2. Gram. Each of the three (or two) grammatical «kinds», corresponding more or less to distinctions of sex or absence of sex [...]. English has «natural» as opposed to «grammatical gender. 3. transf. Sex, now only joc. 4. arch. Offspring.

Avant 1963, le seul sens couramment utilisé était donc le sens deux, à savoir le genre grammatical. Or il ne s'agit pas là d'un concept essentiel à la langue anglaise, dont il faut protéger l'intégrité à tout prix. Sur le 
plan sémantique, gender pouvait donc acquérir sans difficulté un nouveau sens et s'installer dans le champ sans le déstabiliser. Gender, dans son nouveau sens, s'est donc très facilement imposé lorsque la deuxième vague des féministes issues des mouvements socialistes et des droits civiques a imposé une nouvelle manière de regarder la femme. L'usage du terme s'est répandu et l'étude de la femme du point de vue social et culturel a pris le nom de «gender studies», expression qui s'est elle aussi rapidement lexicalisée. Il suffit de regarder les catalogues des grands éditeurs anglophones pour s'apercevoir de la vitalité du domaine d'étude: Routledge par exemple offre 121 titres sous «Gender Studies» (mars 2001). Une recherche sur Internet à l'aide du moteur Google affiche 743000 références en 0,03 seconde pour «Gender Studies» (mars 2001). Les dictionnaires britanniques récents confirment l'usage: le New Oxford Dictionary of English (1998) place le nouveau sens de gender en seconde position (après le sens grammatical) et le Cambridge International Dictionary of English, destiné à l'apprentissage de l'anglais langue étrangère, le place en première position, avant le sens grammatical qu'il qualifie de specialized.

En français, le terme gender dans son sens grammatical se traduit par genre (grammatical) et ne pose normalement pas de problème. Mais le genre grammatical est un concept central à la langue et le terme a en outre une foule d'autres sens (le Petit Robert lui consacre une demicolonne), entre autres celui de «catégorie d'œuvres», que l'anglais traduit par genre - on a ainsi littératures de genre - et celui «d'espèce, sorte» - ce genre de personne. Mais aucun terme français ne répond à la nouvelle définition de gender en anglais. Si l'on examine les solutions offertes par exemple par René Meertens dans son Guide anglais-français de la traduction, d'ordinaire très utile dans de pareils cas, on trouve sous gender: «sexe, différenciation homme-femme, appartenance à un sexe déterminé». L'entrée comporte un grand nombre de traductions de collocations ou expressions lexicalisées (près d'une demi-colonne sur une page comportant deux colonnes). Ces traductions sont utiles dans certains contextes précis mais ne correspondent pas à la définition donnée plus haut. Si l'on se tourne vers des dictionnaires spécialisés, tels que le Dictionnaire de la Psychologie de Werner D. Frölich, édition française de LGF 1997, on lit:

Genre (genus, pl. genera; Gattung), ensemble d'êtres vivants englobant, selon des règles de répartition, une pluralité d'espèces (species, Arten). À leur tour, les genres sont réunis en familles. Dans un sens figuré, le genre désigne un ensemble de faits ou d'événements regroupés selon des caractéristiques déterminées.

Désigne aussi la différenciation entre le masculin et le féminin ( $\rightarrow$ identité de genre). 
Bien que la deuxième partie de la définition soit proche de la définition de gender en anglais, elle ne correspond pas exactement à celle-ci. Elle donne cependant lieu à une expression lexicalisée: identité de genre (voir ANNEXE 1). En cherchant dans d'autres ouvrages de psychologie plus techniques on trouve d'autres expressions lexicalisées telles que schéma de genre, constance de genre, stabilité de genre.

On peut, malgré tout, choisir la voie du néologisme et traduire «gender» dans sa nouvelle acception par «genre». Ceci signifie ajouter un nouveau sens au terme «genre». Mais l'addition de ce nouveau sens - ou s'agit-il d'un homographe homonyme? - déstabilise le champ et entraîne la confusion dans un grand nombre de cas où le contexte devient indispensable pour distinguer les sens. Exemples: étude de genre(s) (gender studies), changement de genre, spécifique au genre, normes $d u$ genre, etc.

Sous la poussée des publications en langue anglaise des recherches en sociologie, anthropologie, etc., les traducteurs et les spécialistes des divers domaines où le concept est utilisé ont été contraints de trouver des solutions ou des équivalences. Les Québécois, en première ligne, ont souvent choisi de traduire gender par genre et gendered par genré. En France de nombreux sociologues font de même. Gaïd Le Maner Idrissi, par exemple, psychologue et auteur de L'Identité sexuelle distingue l'identité sexuelle, l'identité de genre et l'identité sexuée. L'usage de «genre» dans ce sens spécifique semble actuellement restreint à un usage spécialisé, mais il n'est pas sûr qu'il le reste puisqu'on peut commencer à entendre et à lire ici et là des expressions telles que : changement de genre, transgenre, ceux de votre genre, etc.

\section{Gender en contexte}

Reprenons maintenant le texte qui a été le point de départ de la réflexion pour repérer les occurrences du terme. Dans le long texte, qui comporte près de 27000 mots, gender apparaît 123 fois, et dans le texte court de 3650 mots, 20 fois, ce qui exclut toute traduction au coup par coup sans harmonisation. Si l'on regarde de plus près, gender apparaît comme nom ou en position adjectivale dans les groupes ou collocations suivants:

$\begin{array}{ll}\text { Texte court } & \\ \text { gender } & 5 \\ \text { gender } \text { disadvantage } & 1 \\ \text { gender discrimination } & 3 \\ \text { gender } \text { equitable } & 1 \\ \text { gender } \text { equity } & 3 \\ \text { gender } \text { inequities } & 1\end{array}$


gender perspective

gender relations

gender roles

gender sensitisation

gender sensitive

Total:

\section{Texte long}

gender

gender bias

gender biases

gender comparisons

gender difference

gender differences

gender equality

gender equitable

gender equity

gender identities

gender identity

gender imbalance

gender inequities

gender issues

gender normative patterns

gender norms

gender patterns

gender perspective

gender potentials

gender power

gender relations

gender roles

gender scripts

gender socialisation

gender specificity

gender theories

gender violence

gender-related patterns

gender-specific challenges

gender-specific educational challenges

gender-specific health needs

gender-specific perspective

gender-specific potentials

gender-specific socialisation

gender-specific theories

gendered patterns

genderless

intra-gender violence

3

Total:

Un coup d'œil à la liste permet de juger de la cohérence de l'ensemble. Le terme gender structure l'argumentation et les 123 termes relevés dans 
32 des 40 pages du texte tissent un fil conducteur qui guide le lecteur en le renvoyant constamment à l'approche choisie. Il est évident que traduire gender de diverses manières signifie détruire cette cohérence et affaiblir la structure interne du texte en renvoyant le lecteur uniquement aux concepts abstraits et non plus à une unité d'expression. Prenons quelques exemples de traductions possibles (voir ANNEXE 2):

gender equity/equities/inequities: égalité (s)/inégalité (s) entre les hommes

et les femmes, égalité/inégalité homme-femme, égalité/inégalité

entre les sexes

gender differences: différences entre les sexes

gender role: rôle de chaque sexe, rôle sexué

gender pattern: mode de comportement sexué

gender perspective: perspective sexuée

gender specific: propre à chaque sexe, à l'un ou l'autre sexe, sexospécifique

gender socialisation: socialisé selon le sexe

gender relations: relations hommes/femmes, etc.

Gender se trouve exprimé par sexe, sexué ou homme/femme. Cette traduction est acceptable dans un grand nombre de cas. Elle convient pour un francophone, mais n'est pas fidèle sur le fond si l'on se réfère à la définition de gender. Elle pose par ailleurs d'autres problèmes de traduction ayant une incidence sur la cohérence de l'ensemble du texte.

Très logiquement du point de vue de la rédaction du texte, gender apparaît en effet tout au début, en page 4, dans l'introduction:

Applying a Gender Perspective to Adolescent Boys

The «why» of focusing on adolescent boys emerges from a gender perspective. The review of research used a gender perspective from two approaches: gender equity and gender specificity.

Gender equity refers to the relational aspects of gender and the concept of gender as a power structure that often affords or limits opportunities based on one's sex. Gender equity applied to adolescent boys implies, among other things, working with young men to improve young women's health and well-being, and their relative disadvantage in most societies, taking into consideration the power differentials that exist in many societies between men and women.

Il est évident que l'on ne peut avoir recours ici aux solutions envisagées plus haut et qu'il est impossible d'éviter l'introduction du terme «genre» pour traduire le concept de gender, puisque celui-ci est défini dans le texte et que le terme sex apparaît dans la même phrase.

Plus bas, page 11, apparaît le terme «gender-specific theories», repris un peu plus loin par «theories on gender identities». Il s'agit bien entendu des théories fondant le concept de gender, et non du sexe ou de la sexuation.

Enfin, gender et sex s'opposent dans certaines expressions et structurent le découpage sexe naturel/sexe social: gender identity/sexual identity, sexual roles/gender roles et sexual and gender scripts (une seule expression). 
Un examen plus détaillé du texte met en évidence le nombre très élevé des occurrences de sex et sexual: sex/sexes apparaît 112 fois, sexual 199 fois. Les traductions de gender par «sexe, sexuel» dilueraient donc le concept de gender dans le très grand nombre de ces occurrences, qu'il ne sera pas possible de traduire en français autrement que par sexe ou sexuel/le, et effaceraient toute opposition entre sex et gender. La solution qui s'impose est donc le recours à «genre» dans les cas qui viennent d'être cités: définition, articulation de la pensée autour des oppositions gender/sex etc. Toutefois, l'introduction de «genre» pour traduire gender dans certains cas aura des répercussions sur l'ensemble des traductions de gender. La question qui se pose alors est la suivante: faut-il décider de traduire systématiquement gender par genre et gendered par genré? Cela donnerait dans ce cas (voir ANNEXE 2):

gender equity/equities/inequities: égalité (s)/inégalité (s) entre les genres gender differences: différences selon le genre gender role: rôle selon le genre gender pattern: mode de comportement genré gender perspective: perspective genrée gender specific: propre à chaque genre gender socialisation: socialisé selon le genre gender relations: relations entre les genres, etc.

Cette solution n'est pas vraiment satisfaisante. Il est cependant difficile de traduire gender par genre uniquement dans l'introduction et dans trois ou quatre cas spécifiques, et de ne plus réutiliser le terme dans le reste du document. J'ai donc choisi la solution consistant à utiliser de manière plus ou moins équilibrée les traductions: selon le sexe, sexué, homme/femme etc. et genre, de genre, genré (voir ANNEXE 3).

\section{gender toujours traduit par genre}

gender (nom, isolé): genre

gender identity: identité de genre (lexicalisé dans les publications spécialisées)

gender scripts: schémas de genre (lexicalisé dans les publications spécialisées)

\section{gender parfois traduit par genre}

gender specific: propre à chaque sexe, à l'un ou l'autre sexe, propre au genre gender bias: parti pris lié au sexe/parti pris lié au genre

gender issues: questions liées aux spécificités de chaque sexe/au genre gender perspective: perspective sexuée ou perspective genrée

\section{gender traduit autrement que par genre}

gender differences: différences hommes/femmes

gender-specific health needs: besoins de santé selon le sexe, sexospécifiques

Le résultat n'est pas satisfaisant, mais me semble moins insatisfaisant que la traduction systématique de gender par genre. Bien que la cohé- 
rence du texte ne soit pas respectée et que la traduction ne soit pas fidèle à la définition du concept, le texte semble toutefois plus lisible pour un non-spécialiste, critère important puisque le document n'est pas destiné à des spécialistes. La voie moyenne du traducteur est souvent source d'insatisfaction. D'autres traducteurs auraient sans aucun doute fait d'autres choix.

On peut supposer que lorsque la diffusion du concept sera plus large, une traduction s'imposera. Ce sera probablement «genre», qui sera tout simplement importé avec son sens du fait de la proximité des deux langues.

Il reste naturellement des questions, d'ordre sociologique ou culturel, auxquelles je n'ai pas répondu. Pourquoi le concept de gender est-il né dans les pays anglophones et pourquoi s'est-il répandu aussi rapidement et avec un tel succès, prouvant qu'il existait un besoin conceptuel? Pourquoi les pays francophones n'ont-ils par ressenti le même besoin à la même époque? Pourquoi les mouvements féministes français n'ont-ils pas imposé ce concept à la société alors que la France a fourni, outre Simone de Beauvoir, des théoriciennes féministes telles que Hélène Cixous, Luce Irigaray (citées même dans l'Encyclopédie Microsoft Encarta) reconnues outre-Atlantique? La France semble rétive à ce genre (?) d'approche. Pourquoi? Est-ce parce que les rapports hommefemme y sont différents? Parce que les femmes manquent de maturité ou d'indépendance? Pour d'autres raisons? Nous atteignons ici la ligne de partage entre la lexicologie/traductologie et le domaine de la sociologie et des études culturelles.

Avant de terminer, examinons quelques autres difficultés de traduction qui sont intéressantes du point de vue culturel, toujours sur le même thème et à propos du même document. Tout d'abord dating/courtship violence et date rape. Il s'agit de la violence au sein d'un couple de jeunes personnes qui sortent ensemble (habituellement) ou d'un viol commis par un compagnon de sortie. Ces collocations posent deux types de problèmes culturels. Tout d'abord «dating», que le New Oxford English Dictionary (1998) définit ainsi :

«chieflly N. Am., go out with someone in whom one is romantically or sexually interested », exprime une conception nord-américaine de la sortie en couple et n'a pas d'équivalent exact en français. Toutefois, isolé et en contexte, le terme ne pose pas de difficultés. On peut le traduire par avoir rendez-vous avec, avoir un (e) petit (e) ami (e), sortir avec, etc. Mais les collocations dating/courtship violence et date rape sont plus difficiles à traiter. Elles sont surtout nord-américaines et sont lexicalisées. On trouve date rape dans certains dictionnaires et, en cherchant sur 
Internet les expressions dating ou courtship violence, on obtient de nombreuses réponses sur des sites généralistes destinés aux parents, dans les feuilles «Questions fréquemment posées», à côté des questions sur l'acné ou les grossesses précoces. On trouve également des sites plus sérieux, comme ceux du ministère de la Santé des États-Unis ou des Centers for Disease Control and Prevention de l'État de Californie, qui sont bien documentés et qui accompagnent leurs explications d'une longue bibliographie. Les sites en français, en revanche, traitent le problème de la violence de manière différente, plus globale. Les sujets abordés sont ceux de la violence familiale et conjugale. Le contexte de la violence au cours de sorties en couple n'est pas abordé. La traduction de ces expressions impose donc une précision sur le contexte qui semble inutile, superflue ou hors de propos en français, comme par exemple «les femmes victimes d'un viol commis par leur compagnon lors d'une sortie en couple». Le problème posé par ce type de violence agite les campus américains depuis des années, mais le contexte français, général ou universitaire, est trop différent pour que le problème puisse être posé dans les mêmes termes. La difficulté est donc bien d'ordre culturel. Dans le cas présent d'un document destiné à un lectorat international, on peut légitimement se poser la question du bien fondé du traitement de ce type de problème.

Enfin, le dernier point particulier est celui des nombreux euphémismes et de l'approche féministe que j'appellerai «jusqu'au-boutiste», lesquels relèvent aussi de la culture. Le document contenait en effet un certain nombre d'euphémismes intraduisibles tels quels, par exemple: $M S M=$ «men who have sex with other men », c'est-à-dire homosexuels, pour éviter ce terme qui véhicule des préjugés, same sex relationship, expression lexicalisée, pour relation homosexuelle, sex worker pour prostitué(e), bien que travailleur/travailleuse du sexe ait peut-être un avenir en français. Il contenait aussi deux termes «ménarche» (apparition des premières règles) et «spermarche» (première spermatogenèse) présentant un intérêt. Le terme ménarche figure en effet dans les dictionnaires spécialisés, mais spermache est peu usité, difficile à trouver même dans les dictionnaires techniques, et son genre fluctue: c'est parfois un nom féminin, parfois un nom masculin. Le recours à ce terme rare a pour origine un parti pris égalitariste homme/femme. Il fallait trouver un équivalent au processus physiologique de la ménarche chez les jeunes filles. La spermache fait donc pendant à la ménarche. Ces termes techniques ne posent pas de problèmes de traduction mais le parti pris qui a justifié leur choix peut être ressenti par le lecteur comme culturellement très éloigné.

Il n'est pas surprenant que des textes touchant à la société posent des problèmes de traduction puisqu'une société imprime sa marque sur ses 
membres et que l'ensemble des formes acquises de comportement constitue la culture. Parler de problèmes de société, c'est parler de culture. Traduire des textes pragmatiques dans le domaine de la sociologie et des sciences annexes, c'est tenter de jeter des ponts entre les cultures pour que le sens puisse passer, permettant à la communication interculturelle de s'établir.

\section{Ouvrages cités dans le texte}

Ayto, John, 20th Century Words, Oxford, Oxford University Press, 1999. Frölich, Werner D., Dictionnaire de la Psychologie, Paris, Encyclopédies d'aujourd'hui, édition de 1997.

Le Maner-Idrisssi, Gaïd, L'Identité sexuée, Paris, Dunod, 1997.

Meertens, René, Guide anglais-français de la traduction, Paris, Top éditions, 1999.

Petit Robert (Le), Paris, SNL, édition de 1996.

The New Oxford Dictionary of English, Oxford, Oxford University Press, 1998.

The Shorter Oxford English Dictionary, Oxford, Oxford University Press, Éditions en deux volumes, 1973.

\section{ANNEXE 1}

\section{Définitions}

Twentieth Century Words John Ayto (OUP, 1999)

Gender, 1963:

The sex of a human being, from the point of view of the social and cultural, as opposed to the biological, distinctions between the sexes. Often used as a noun.

Alex Comfort:

The gender role learned by the age of two years is for most individuals almost irreversible, even if it runs counter to the physical sex of the subject. (p. 408).

Shorter Oxford 1973 (3 éd.)

Gender

1. kinds 2. Gram. Each of the three (or two) grammatical «kinds», corresponding more or less to distinctions of sex or absence of sex [...]. English has «natural» as opposed to grammatical gender. 3 . transf. Sex, now only joc. 4. arch. Offspring. 
Dictionnaire de la Psychologie, Werner D. Frölich, édition française LGF, 1997 :

Genre

genre (genus, pl. genera; Gattung), ensemble d'êtres vivants englobant, selon des règles de répartition, une pluralité d'espèces (species, Arten). À leur tour, les genres sont réunis en familles. Dans un sens figuré, le genre désigne un ensemble de faits ou d'événements regroupés selon des caractéristiques déterminées.

Désigne aussi la différenciation entre le masculin et le féminin (->identité de genre).

\section{Identité de genre}

Gaïd L Maner-Idrisi, Identité sexuelle, Dunod 1997.

L'identité de genre fait référence au sexe social et psychologique. À l'inverse de l'identité sexuelle, l'identité de genre exclut la dimension sexuelle, c'est-à-dire tout ce qui a trait à la sexualité et aux relations qu'elle implique entre les sexes. Plus précisément, Unger définit l'identité de genre comme suit:

Le terme genre peut être utilisé pour désigner les composantes non physiologiques du sexe qui sont actuellement perçues comme appropriées aux individus de sexe masculin ou aux individus de sexe féminin.

R.K. Unger, Female and Male: Psychological Perspective, New York, Harper \& Row, 1979.

\section{Schéma de genre}

Gaïd L Maner-Idrisi, Identité sexuelle, Dunod 1997.

... afin de rendre compte de l'organisation cognitive nécessaire à la mise en place des conduites appropriées au sexe, Martin et Halverson (1981), ont introduit la notion de schéma de genre.

... à la fin de la deuxième année l'enfant dispose d'un système de référence interne relatif aux catégories sociales du sexe (un schéma de genre). 


\section{ANNEXE 2}

\section{Gender traduit autrement que par genre}

gender equity/equities/inequities : égalité (s)/inégalité (s) entre les hommes et les femmes, égalité homme-femme, égalité entre les sexes gender differences: différence entre les sexes gender role: rôle de chaque sexe, sexué gender pattern : mode de comportement sexué gender perspective: perspective sexuée gender specific: propre à chaque sexe, à l'un ou l'autre sexe, sexospécifique gender socialisation : socialisé selon le sexe gender relations: relations hommes/femmes

\section{Gender traduit par genre}

gender equity/equities/inequities : égalité (s)/inégalité (s) entre les genres

gender differences: différences selon le genre gender role: rôle selon le genre gender pattern : mode de comportement genré gender perspective : perspective genrée gender specific: propre à chaque genre gender socialisation: socialisé selon le genre gender relations: relations entre les genres

\section{ANNEXE 3}

Extrait 1: Gender et sex apparaissant simultanément dans le même passage

Anglais

Applying a Gender Perspective to Adolescent Boys

The «why» of focusing on adolescent boys emerges from a gender perspective. The review of research used a gender perspective from two approaches: gender equity and gender specificity.

Gender equity refers to the relational aspects of gender and the concept of gender as a power structure that often affords or limits opportunities based on one's sex. Gender equity applied to adolescent boys implies, among other things, working with young men to improve young women's health and well-being, and their relative disadvantage 
in most societies, taking into consideration the power differentials that exist in many societies between men and women.

\section{Français}

Appliquer aux garçons adolescents la grille de lecture du genre. Pourquoi s'intéresser aux garçons? Les raisons en sont évidentes lorsque l'on applique la grille de lecture de la perspective sexuée ou du genre, c'est-à-dire du partage établi des rôles hommes/femmes. Le présent tour d'horizon des recherches a fait appel à cette grille à partir de deux approches : égalité entre les sexes et spécificité des sexes. L'égalité entre les sexes renvoie aux aspects relationnels du genre et au concept de genre en tant que structure de pouvoir, qui souvent offre des possibilités ou limite celles-ci selon le sexe. Rechercher l'égalité entre les sexes dans le cas des garçons adolescents signifie entre autres travailler avec les jeunes gens afin d'améliorer la santé et le bien-être des jeunes femmes et lutter contre les discriminations dont celles-ci font l'objet dans la plupart des sociétés, compte tenu du différentiel de pouvoir qui existe entre les hommes et les femmes dans de nombreuses sociétés.

Extrait 2: Identité de genre

\section{Anglais}

Boys and Gender Identity Formation

These new perspectives on adolescent males build on previous research and theories on gender identity development and gender socialisation during early childhood. Many developmental psychologists argue that fundamental aspects of gender identity are linked to the earliest experiences of being cared for and to the person giving that care. According to these theories, the fundamental task of early gender identity development for boys is to develop a separate gender identity than the mother's and thus achieve a greater normative separation from the mother than girls generally do. At the stage of separation from the primary attachment figure (generally the mother), a boy must achieve separation and individuation, and publicly define his gender identity (Gilmore, 1990; Chodorow, 1978).

\section{Français}

Les garçons et la formation de l'identité de genre.

Les nouvelles perspectives appliquées aux adolescents de sexe masculin présentées ici reprennent et développent les recherches et les théories déjà publiées sur la construction de l'identité de genre et la 
socialisation sexuée au cours de la petite enfance. De nombreux psychologues du développement estiment que les aspects fondamentaux de l'identité de genre sont liés aux toutes premières expériences de l'enfant lorsque celui-ci fait l'objet de soins, ainsi qu'à la personne qui dispense ces soins. Selon ces théories, le rôle fondamental des premières étapes du développement de l'identité de genre chez les garçons est de développer une identité de genre distincte de la mère et de parvenir ainsi à une séparation normative d'avec la mère plus marquée que chez les filles. A ce stade de la séparation d'avec la personne avec qui s'est nouée la relation d'attachement primaire (généralement la mère), le garçon doit parvenir à la séparation et à l'individuation, et définir publiquement son identité de genre (Gilmore, 1990; Chodorov, 1978). 